\title{
Culture, styles de management, GRH et performance des organisations marocaines : Revue de littérature
}

\author{
RAJÂA Omar ${ }^{1}$ \\ ${ }^{1}$ Enseignant Chercheur à l'Ecole Nationale de Commerce et de Gestion \\ Université Ibn Zohr - Maroc
}

Résumé : Cette revue de littérature nous a permis de mieux appréhender le contexte marocain pour pouvoir comprendre le rôle des pratiques RH dans la performance des organisations marocaines et d'exposer les principaux résultats des études effectuées dans ce sens.

Mots-clés : GRH, Styles de Management, Performance des organisations et Culture.

Notre article dans sa quête d'objectivité et surtout d'apporter une valeur ajoutée à l'édifice de la connaissance dans les domaines respectifs de la GRH, de la culture et de la performance des organisations, nous avons cherché, via l'analyse des différentes études effectuées au Maroc, à comprendre, et obtempérer aux mécanismes d'interrelation entre les pratiques RH et la performance des organisations marocaines.

\section{Culture et styles de management au Maroc}

Koullou ${ }^{1}$ avait stipulé qu'il existait au Maroc une culture en mosaïque avec plusieurs sous cultures, mais qui peuvent être ramenées aux valeurs suivantes : la logique d'allégeance, le familisme et la sacralité de la dignité. La mise en œuvre de ces valeurs dans une perspective de gestion donne lieu à l'apparition de nombreux types et modes de gestion qui caractérisent l'entreprise marocaine d'aujourd'hui. Cependant, des constantes semblent toutefois apparaître.

Selon les dimensions de la fameuse étude de Hofstede ${ }^{2}$, on peut relever les caractéristiques du management marocain comme suit :

\footnotetext{
${ }^{1}$ Larbi Koullou (« in la vie économique », Août 1991)

${ }^{2}$ Hofstede G. : «Vivre dans un monde multiculturel : comprendre nos programmations mentales », Les Editions d'Organisation, Paris 1994.
} 
(2e management marocain avait un score de 70 points, au même niveau que celui de pays comme l'Egypte ou la France, ce qui conduisait, au niveau des organisations, à des systèmes centralisés, des liens de subordination confirmés et des profils de manager à tendance autocratique.

- La société marocaine est considérée comme moyennement individualiste, c'est-à-dire que les engagements et la loyauté au groupe, à la famille ou à la communauté en général conditionnaient les actions et comportements. Cette situation avait fait que la relation de l'employeur à l'employé (en matière de motivation des équipes, de résolution des conflits ou de conduite du changement...) était perçue comme un lien moral avant tout.

- La société marocaine était moyennement masculine avec un score de 53 points, comparée à l'Egypte (45 points) ou la France (43 points). Ce qui pouvait expliquer, selon l'auteur, la tendance des entreprises à opter pour des démarches de type RSE (Responsabilité sociale de l'entreprise) ainsi que des phénomènes comme le stress au travail ou les conflits au sein des entreprises.

- Le score lié à la maîtrise de l'incertitude, qui était relativement faible par rapport à celui de l'Egypte ou celui de la France et plus important que celui de la Finlande, faisait que le marocain avait moins de propension à contrôler le temps et serait dans une logique de laisser-aller par rapport à la France et l'Egypte. Cette constatation pouvait, selon l'auteur, expliquer des comportements constatés au sein des entreprises marocaines, comme le respect des engagements, la ponctualité et la rigueur dans la planification des projets et actions futures.

En matière de pragmatisme, la société marocaine était loin derrière les pays occidentaux quoique relativement meilleurs que l'Egypte. Ce constat pouvait être expliqué d'une part, par le fait que les marocains étaient attachés au respect des traditions et portés sur l'explication et moins sur les réalisations et d'autre part, par la tendance du management marocain à être moins focalisé sur le résultat et à privilégier le retour immédiat au lieu d'une orientation sur le long terme. 
$\rightarrow \quad \mathrm{Au}$ niveau de l'indulgence comme valeur partagée, le Maroc avait un score moins important que les pays occidentaux mais nous restons mieux placés que des pays comme 1'Egypte. Avec des caractéristiques comme la prédominance des comportements impulsifs non rationnels et des propensions importantes au cynisme et au pessimisme, les entreprises seraient un terrain fertile pour les conflits, les tensions et les pratiques qui s'écartaient des codes éthiques et déontologiques reconnus.

Figure 1 : Synthèse Comparative des six dimensions de Hofstede Maroc, Egypte, France et Finlande.

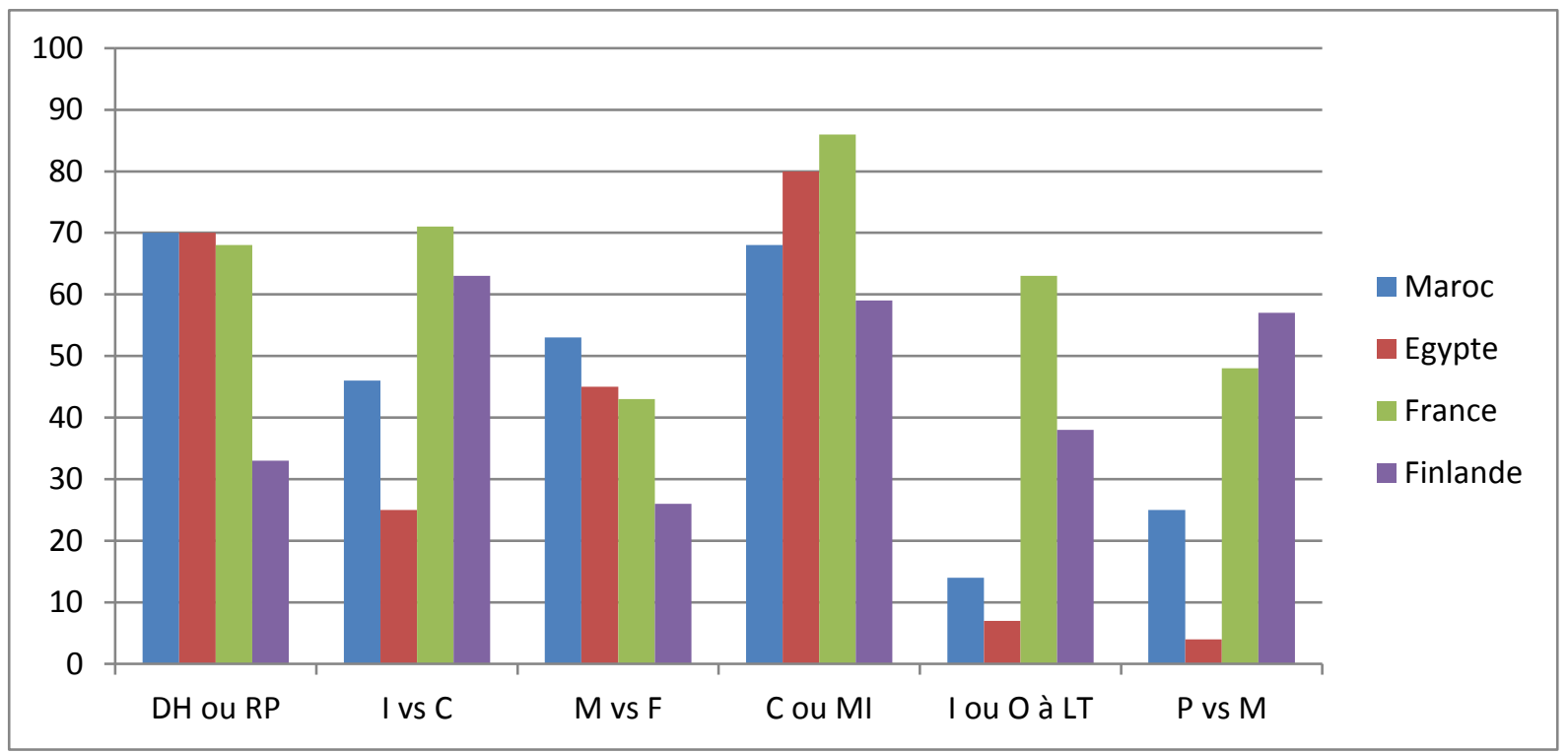

Pour compléter cette analyse de la place de la culture dans le management marocain et surtout pour l'actualiser, on va exposer dans ce qui suit, les principales études effectuées dans ce sens :

En 2003, El Amrani et Chebihi $^{3}$, partant du constat sur la relative universalité de la dynamique organisationnelle et des questionnements sur l'influence de l'environnement culturel marocain sur le management des organisations et en menant des entretiens auprès de 12 cadres marocains, avait cherché à comprendre comment être sensible aux facteurs culturels et à quel niveau, pour une pratique managériale efficace. Les résultats de cette étude avaient attesté que les dirigeants et cadres marocains exprimaient les qualités normatives de la culture marocaine ${ }^{4}$ par le biais de valeurs qu'ils privilégiaient

\footnotetext{
${ }^{3}$ El Amrani J. et Chebihi M.A., «Culture et management au Maroc », Repères et perspectives, Rabat, n 4, hiver, 2003 , p. 87-107.

${ }^{4}$ Les caractéristiques de la culture marocaine prises en considération étaient : L'Islam Malékite, les langues arabes et berbères, la famille, l'ethnie,

l'éducation et l'aspiration à la modernité.
} 
dans leur mode de management. Les auteurs avaient regroupé ces valeurs dans deux catégories :

$\checkmark \quad$ Des valeurs culturelles qui véhiculaient une vision largement partagée au sein de la société marocaine et surtout elles avaient un «sens» pour la majorité des marocains (Solidarité et attachement à l'ethnie) ;

$\checkmark \quad$ Des valeurs qui condamnaient les entreprises marocaines à rester soumises à la fatalité et à rater le train du changement et du progrès (Distance hiérarchique, domination des valeurs masculines, perception sexiste de la femme, vision à court terme, culture d'ostentation, opportunisme et culture de la rente).

En 2007, l'étude de Benabdeljli1 ${ }^{5}$, qui était menée auprès d'un échantillon de responsables hiérarchiques d'entreprises industrielles marocaines, avait analysé l'influence de la culture sociétale sur les relations hiérarchiques et les attitudes managériales. Les résultats avaient révélé qu'une entrée par la culture était utile mais insuffisante pour comprendre les spécificités des attitudes au travail et des relations sociales dans l'entreprise. D'autres variables contextuelles explicatives surtout celles qui conditionnent le degré de précarité des situations de travail dans les PME marocaines étaient mises en évidence. L'étude avait aussi mis évidence comment les leviers culturels étaient mobilisés par certains managers au Maroc pour asseoir leur pouvoir.

En 2014, Belalia ${ }^{6}$, en se tournant vers la grille d'analyse de l'impact de la culture nationale, sur les styles de management au sein des entreprises ${ }^{7}$, avait tiré trois enseignements concernant le management marocain :

L'environnement socioculturel influençait fortement les styles de management : les facteurs historiques et culturels qui nous lient à la France expriment en grande partie les scores comparables, notamment pour la répartition du pouvoir et la tendance à privilégier ou pas la performance.

\footnotetext{
${ }^{5}$ Benabdeljlil N. : «Les modes de management des entreprises au Maroc : entre contingences culturelles et économiques », Revue internationale P.M.E., Vol. 20, № 2, 2007, pp. 89-122.

${ }^{6}$ Belalia A. : «Existe-t-il un management marocain ? », L’Economiste, Edition № 4398, Le 12/11/2014

${ }^{7}$ Notamment celle de Hofstesde déjà signalée en introduction générale.
} 
La dimension culturelle arabo-musulmane aurait des effets sur les modes de gestion comparables à ceux des pays comme l'Egypte avec lesquels le Maroc avait des scores comparables en matière de répartition de pouvoir, de masculinité et de pragmatisme.

$\checkmark \quad$ En s'ouvrant de plus en plus sur le modèle capitaliste dominant, le Maroc serait plus porter vers des valeurs comme le culte de la performance, le triomphe de l'individualisme et l'affirmation des disparités hiérarchiques et sociales.

En 2014, Azouzou ${ }^{8}$ avait stipulé que les facteurs culturels de compétitivité au Maroc sont: la culture chevaleresque et les valeurs de dignité et de solidarité. Les deux premières sont indispensables pour le dépassement de soi (besoin d'accomplissement de Maslow), la troisième est indispensable pour le management de groupe (cercles de qualité, groupes amélioration qualité). L'auteur avait constaté que le potentiel culturel national n'est, cependant, pas encore suffisamment mobilisé au service du développement pour un épanouissement des composantes du trinôme : société, groupe et individu.

En 2015, L'étude de Benabdeljlil' ${ }^{9}$, qui était menée auprès de responsables hiérarchiques de 35 entreprises de divers secteurs ${ }^{10}$, s'était interrogée sur le rôle des facteurs culturels dans les modes de management des entreprises marocaines ${ }^{11}$ et dans quelle mesure la culture influence-t-elle les comportements et les modes de réponse à des situations données. Les situations de travail qui étaient étudiées avaient révélé des constantes culturelles (investissement affectif dans les relations hiérarchiques plus personnalisées, tendance au respect de la hiérarchie, voire à la soumission à l'autorité et à un amourpropre de la main-d'œuvre au travail) et des variables contextuelles (le faible niveau de qualification de la main-d'œuvre et la situation structurelle de crise sur le marché de l'emploi) qui généraient chez les ouvriers marocains des comportements rationnels

\footnotetext{
${ }^{8}$ Azouzou H. : «La dimension culturelle de la qualité », Albayane, le $05-01-2014$.

${ }^{9}$ Benabdeljlil N. : «Rapports sociaux et attitudes au travail dans les entreprises marocaines : entre contingences culturelles et contingences économiques », In Dupret et al. (dir), Le Maroc au présent, Ed. Dialogue des deux rives, 2015, pp. 985-994.

${ }^{10}$ Quasiment tous les secteurs étaient touchés par cette études : Textile-confection, industrie métallurgique et mécanique, industrie agro-alimentaire, ameublement, électroménager, chimie-parachimie, imprimerie, BTP, etc.

${ }^{11}$ Les modes de management étaient analysés à trois niveaux : Rapports hiérarchiques, Régulation du pouvoir ET Attitudes au travail.
} 
avant d'être culturels. En résumé, on peut synthétiser les résultats de cette étude comme suit :

La discipline, comme respect des règles formelles de présence et non comme le respect de procédures de travail, constitue une stratégie rationnelle de la maind'œuvre marocaine. Le chercheur avait attesté que le sens de la discipline était perçu par la plupart non pas comme une valeur intrinsèque aux ouvriers, mais par le fait que cela fait partie des règles du jeu et que ces derniers n'avaient pas le choix ;

Le respect envers les supérieurs se situait entre d'une part une rationalité contingente «crainte », ou « peur» et non reconnaissance d'un statut ou d'une compétence et d'une autre part une « soumission » de type culturel. L'auteur avait affirmé dans ce sens que la culture marocaine, avec des facteurs discriminants relatifs à la personnalité ou à la culture individuelle du manager, était mobilisée par le management au sens où elle était porteuse de valeurs de respect de la hiérarchie et de l'autorité ;

Le sens du devoir était contingent aux situations de travail. Selon les résultats de cette étude les perceptions concernant cet aspect étaient assez variées, et deux groupes pouvaient être distingués. Pour les uns, le sens du devoir était perçu par les ouvriers plutôt négativement à cause soit de leur « niveau intellectuel / niveau de formation » soit du fait que le travail était perçu comme peu valorisant ou encore le facteur technologie et, notamment, la pression du rendement qui semblaient induire des comportements de résistance des ouvriers. Pour les autres, en insistant sur la responsabilité de l'encadrement quant au comportement de la main-d'œuvre, mettaient en avant des stimulants d'ordre culturel («sens du devoir » à des cultures régionales qui seraient porteuses de sens de l'honneur, de dignité), pour mobiliser le personnel avec une perception positive du sens du devoir au travail ;

L'affectivité dans les relations de travail variait entre profil culturel et opportunisme de l'encadrement. Les responsables, en admettant que les aspects 
affectifs jouent «beaucoup » dans les rapports qu'ils avaient avec leurs subalternes, mettaient en œuvre des pratiques de proximité et de valorisation, verbales ou matérielles, avec pour objectif implicite de développer à la fois l'attachement et l'adhésion du personnel. Cependant, l'affectif ne devait pas impliquer le «copinage » car selon une grande majorité des répondant « être gentille, c'est être faible ». En outre, les résultats de cette étude avaient mis en avant que la place occupée par les aspects affectifs paraissait toutefois diminuer avec le degré de formalisation du système managérial qui, dans certaines grandes entreprises, «professionnalise » les relations et rigidifie les rapports humains. Dans ces cas, le degré d'attachement de la main-d'œuvre à l'entreprise était estimé «faible » par les responsables et se faisait d'abord sur les aspects matériels et les conditions de travail ;

Les managers de proximité basculaient d'une confiance déclarée à un contrôle affiché, ce qui rendait la confiance et la pratique du contrôle deux choses indépendantes. Les résultats de cette étude avaient, en outre, montré que les opinions sur la possibilité de faire confiance aux ouvriers étaient plutôt positives et que la possibilité de faire confiance était liée à des représentations positives du sens de la discipline, du respect de la hiérarchie et du sens du devoir. En plus, selon toujours les résultats de cette étude, bien que le contrôle était généralement standardisé et accepté par la main-d'œuvre, ce contrôle posait problème lorsqu'il s'agissait de remettre en question la façon de travailler ;

Les perceptions des responsables hiérarchiques de la capacité d'initiative étaient plutôt négatives. Selon les résultats de cette étude, le manque d'initiative étaient d'ordre culturel: La formation de base et le système d'apprentissage subis induisaient une attitude «passive » qui était accentuée par le cercle vicieux du paternalisme adopté par la majorité des managers de proximité. En effet, ces perceptions renforçaient les tendances managériales à la centralisation, au contrôle, à l'absence de délégation, etc., ce qui entrainait en retour une faible tendance à la prise d'initiatives chez la main-d'œuvre. 


\section{La fonction RH au Maroc : état des lieux et effets sur la performance}

\section{Pratiques RH : Etat des lieux}

En ce qui concerne, la fonction $\mathrm{RH}$, de manière spécifique, elle a pendant longtemps été considérée $\mathrm{au}$ sein des différentes structures organisationnelles du tissu économique marocain comme secondaire et ne constituait souvent pas un élément stratégique. Les chercheurs dans le domaine des $\mathrm{RH}$ au Maroc s'accordent pour affirmer que cette dimension du management de l'entreprise est encore à un stade embryonnaire. "La conception de "l'homme coût » est largement diffusée et fait la règle dans beaucoup de structures. En revanche, on note une prise de conscience de certains chefs d'entreprises de l'importance du capital humain comme sources d'avantage et gisement de richesse.» ${ }^{12}$

De ce fait, la réflexion a été engagée autour de la nécessité d'intégrer la GRH au cœur de la stratégie de développement des administrations publiques et des entreprises privées en prenant en compte leurs spécificités et leurs tailles. Mais, la fonction RH a malheureusement évolué à deux vitesses, principalement à cause des différentes perceptions des dirigeants et des managers à l'égard de la GRH. En effet, on trouve des structures qui continuent à se contenter du strict minimum de la fonction RH, pendant que d'autres développent des politiques plus ambitieuses afin de gérer au mieux les relations entre décideurs, dirigeants, salariés et partenaires sociaux (Bellal ${ }^{13}$ et El Asri $^{14}$ ). Mais, de manière générale, les deux enquêtes réalisées par le cabinet DIORH ${ }^{15}$ en 2002 et 2004 sur la fonction RH dans plus de 100 entreprises ont fait ressortir des évolutions rapides des politiques et pratiques RH et l'adoption de grappes de pratiques proches de celles des entreprises européennes surtout dans les filiales de multinationales. ${ }^{16}$

Pour appuyer ce constat, Plusieurs études ont été menées dans ce sens, une d'entre elles a été réalisée auprès de 73 entreprises industrielles marocaines, dont 70\% sont des PME, et avait porté sur le degré d'élaboration des pratiques de la gestion RH. Cette étude a démontré que dans plus de la moitié des $\mathrm{PME}$, les pratiques $\mathrm{RH}$ restent à un niveau rudimentaire et/ou moyen avec des

\footnotetext{
12 Chaouki F. et Messoudi A. : «Les ressources humaines dans le modèle de performance des PME. Etude des Difficultés et des Besoins d'amélioration de la GRH dans les entreprises marocaines de petite et moyenne taille », Journal Ouest-Africain des Sciences de Gestion $\mathrm{V}_{\mathrm{ol}} .1$, $\mathrm{N}^{\circ}$ 2, 2016, pp. 168-192.

${ }^{13}$ Bellal E. : «'évolution de la gestion des ressources humaines au Maroc », colloque maghrébin sur les Systèmes de formation, stratégies d'entreprises et valorisation des ressources humaines; Faculté de droit de Rabat, 1994.

${ }^{14}$ El Asri L. : «Les pratiques de la gestion des ressources humaines : cas des grandes entreprises au Maroc », International Journal of Innovation and Scientific Research, Vol. 20, N², Feb. 2016, pp. 453-463.

${ }^{15}$ DIORH : «Enquête sur la fonction Ressources Humaines au Maroc », DIORH, Casablanca, 2002, 2004.

${ }^{16}$ Frimousse S. et Peretti J-M. : «Apprentissage Stratégique Des Pratiques De GRH, Internationalisation Des Firmes Et Espace Euromaghrébin », 2005.
} 
résultats qui placent la gestion de carrières en tête des pratiques les moins appliquées, suivie par l'évaluation de la performance et la stratégie de rémunération. ${ }^{17}$

Une autre étude, effectuée en 2013 par Rafai A., a pu constater que la fonction RH dans l'administration se caractérise par l'application impersonnelle de normes et de procédures, abstraction faite du profil des agents, des emplois à pourvoir et des objectifs du service. La GRH, selon toujours cette même étude, se développe par des actes de gestion très classiques, telle l'organisation des concours de recrutement, la notation, les affectations, les mutations, les promotions et les sanctions ${ }^{18}$. Limitée à ce niveau restreint de gestion administrative, la fonction $\mathrm{RH}$ reste le plus souvent une affaire d'hiérarchie dans le secteur public malgré les efforts fournis durant les dernières années afin de la moderniser. En adoptant notamment une approche stratégique du management des RH combinée au concept du New Management Public qui se base sur l'idée que les méthodes du management du secteur privé sont supérieures à celles du secteur public et peuvent lui être transposées ${ }^{19}$.

En 2016, 1'étude de Chaouki, F. et Messaoudi, A. ${ }^{20}$, avait permis de constater que l'état des pratiques RH au sein des PME marocaines dénote d'un besoin manifeste d'une mise à niveau sérieuse de cet aspect crucial de l'organisation. Les affirmations des patrons d'entreprises étaient favorables au redéploiement des activités RH. La conviction que les hommes font la différence de performance est acquise chez un grand nombre d'entre eux bien qu'elle ne soit pas aussi évidente. $»^{21}$.

Au-delà de cet angle de vision purement descriptif de l'état des pratiques RH dans le tissu socioéconomique marocain, d'autres études académiques ont cherché ont cherché à étudier des modèles et des relations déjà expérimentées dans d'autres contextes et pays.

Parmi les études qui ont pris le challenge de tester le lien entre les pratiques RH et la performance au sein des organisations marocaines, nous pouvons citer celle effectuée en 2015 par El Adraoui $\mathrm{H}^{22}$. Cette étude effectuée auprès de 92 entreprises marocaines, a permis, à travers un modèle configurationnel, d'étudier les effets de la GRH sur la performance liée à la rentabilité. Les résultats

\footnotetext{
${ }^{17}$ Guergachi A., Gueye A., Bentaleb C. et Ferar D. : «Le management des ressources humaines au Maroc, quelles pratiques performantes pour les entreprises marocaines?», A ctes du Colloque international La Gestion des Ressources Humaines dans les PME marocaines : quelles pratiques pour la performance ?, Casablanca, 15 et 16 Mars 2013.

${ }^{18}$ Rafai A. : «La gestion des ressources humaines dans la fonction publique au Maroc », Edition 2013.

${ }^{19}$ Huteau S. : «Le management public territorial », Editions du Papyrus Montreuil, 2007.

${ }^{20}$ Chaouki F. et Messoudi A. : «Les ressources humaines dans le modèle de performance des PME. Etude des Difficultés et des Besoins d'amélioration de la GRH dans les entreprises marocaines de petite et moyenne taille », Journal Ouest-Africain des Sciences de Gestion Vol.1, $\mathrm{N}^{\circ}$ 2, 2016, pp. 168-192.

${ }^{22}$ El Adraoui H. : «Effets de la GRH sur la performance: validation du modèle configurationnel auprès d'un échantillon de 92 entreprises au Maroc », Question (s) de management, Vol. 3, 2015, pp. 43-56.
} 
de ce travail attestent, d'un côté, que plus les pratiques RH développées par les entreprises sont cohérentes entre elles, meilleures sont les performances liées à la rentabilité. D'un autre côté, cette étude a pu conclure que l'effet regroupé des pratiques RH explique davantage la performance des grandes entreprises au Maroc que ne peut le faire la somme des effets individuels de ces pratiques.

Ce bref regard rétrospectif sur les pratiques $\mathrm{RH}$ et plus haut le rôle de la culture dans les styles de management au Maroc, nous amène à s'interroger sur l'éventuel impact du patrimoine culturel marocain sur la compétitivité et la performance des entreprises marocaines.

\section{Effet contingent de la culture dans la relation pratiques RH et performance des entreprises marocaines}

L'illustration de l'effet contingent de la culture dans la relation pratiques RH et performance des entreprises marocaines est reconnue principalement aux études suivantes :

- En 2014, L'étude d'Azouzou ${ }^{23}$ avait cherché à déterminer si la variable culturelle pouvait expliquer certains comportements des salariés marocains et comment mobiliser ces derniers pour gérer la qualité au bénéfice de l'entreprise et partant celui de la société. Selon l'auteur, les facteurs culturels de compétitivité sont à prospecter dans les ingrédients suivants : les valeurs la culture chevaleresque, de dignité et de solidarité. Les deux premières sont indispensables pour le dépassement de soi (besoin d'accomplissement de Maslow), la troisième est indispensable pour le management de groupe (cercles de qualité, groupes amélioration qualité). Au final, l'auteur s'était forcé de constater que le potentiel culturel national n'est, cependant, pas encore suffisamment mobilisé au service du développement pour un épanouissement des composantes du trinôme : société, groupe et individu.

- En 2015, l'étude de Moutaouakil ${ }^{24}$, qui était menée auprès de 70 grandes entreprises marocaines, avait comme objectif de vérifier empiriquement l'impact du Contrôle de Gestion Sociale sur la performance économique. Les résultats de cette étude avaient indiqué que, plus l'entreprise est performante socialement, plus elle le sera sur le plan économique et que la majorité des pratiques RH avaient un impact positif sur la performance individuelle et collective. En plus, un système de Contrôle de Gestion

\footnotetext{
${ }^{23}$ Azouzou H. : «La dimension culturelle de la qualité », Albayane le $05-01-2014$

${ }^{24}$ Moutaouakil A. : «L'impact du Contrôle de Gestion Sociale sur la Performance Economique : Cas des Grandes Entreprises Marocaines », Thèse pour l'obtention de Doctorat en Sciences Economiques et Gestion, soutenue en 2015 à la FSJES Université Sidi Mohamed Benabdellah - Fès.
} 
Sociale s'était avéré pertinent dans une démarche de pilotage social des grandes entreprises marocaines.

$\rightarrow \quad$ En 2015, L'étude de Laalou et El Guermai25 ${ }^{25}$ 'était penchée sur une vision quantitative des pratiques RH, sur le degré d'implantation des pratiques RH et sur l'impact de cellesci sur la performance d'une société spécialisée dans l'embouteillage d'eau « Société Source Marrakech ». En faisant appel à des indicateurs d'évaluation des principales pratiques RH (Emploi, Appréciation du personnel, Formation continue, GPEC et Développement des $\mathrm{RH}$ ) et l'influence de celle-ci sur la performance financière, économique et sociale de l'entreprise.

- En 2017, Maghni A. et Amine A., ont cherché à travers leur étude ${ }^{26}$ de mesurer l'effet de la socialisation des nouvelles recrues d'une banque marocaine sur leur engagement. $\mathrm{Au}$ final, cette étude a permis de mettre en évidence l'existence d'un effet positif et significatif des tactiques sociales sur l'engagement organisationnel, tant au niveau de l'engagement calculé qu'au niveau de l'engagement affectif. Les interactions avec les membres expérimentés améliorent l'intégration sociale des nouvelles recrues, d'autant plus que leur identité individuelle est respectée. «En effet, lorsque la nouvelle recrue a de bons rapports avec ses collègues et ses supérieurs, et qu'elle est bien informée de ce qu'elle doit faire pour être appréciée positivement, cela ne peut avoir que des effets positifs sur l'engagement, tant que les tactiques organisationnelles mise en ouvre sont de nature sociale $\gg^{27}$.

$\rightarrow \quad$ En 2018, dans leur ouvrage, Dlimi et Alami ${ }^{28}$ avaient stipulé que le maintien de la performance des équipes n'est pas une chose aisée dans le cadre de la PME marocaine et surtout face à un environnement perturbé et sous haute pression concurrentielle. Les auteurs avaient soutenu que la recherche du compromis entre les acteurs peut s'avérer coûteuse pour ces organisations et ils ont présenté une vision sur les méthodes et

\footnotetext{
${ }^{25}$ Laalou A. et El Guermai L. : «Mesurer l'impact du Management des Ressources Humaines sur la Performance de l'entreprise : Cas de la société Source Marrakech », International Journal of Innovation and Applied Studies, Vol. 13, № 2, 2015.

${ }^{26}$ Maghni A. et Amine A. : «Socialisation Organisationnelle Et Engagement Des Nouvelles Recrues D'une Banque Marocaine : Etude Des Effets Spécifiques Par La Méthode PLS », Revue Marocaine de Management Logistique et Transport, №2, 2017, pp. $239-272$.

${ }^{27}$ Maghni A. et Amine A. : «Socialisation Organisationnelle Et Engagement Des Nouvelles Recrues D'une Banque Marocaine : Etude Des Effets Spécifiques Par La Méthode PLS », Revue Marocaine de Management Logistique et Transport, №2, 2017, pp. 239-272.

${ }^{28}$ Dlimi S. et Alami F-Z. : «Performance des équipes : cas des PME marocaines », Editions L'Harmattan, 2018.
} 
stratégies permettant de garantir la performance durable des équipes particulièrement au sein des PME familiales marocaines.

- En 2019, l'étude de Yassine et Côme ${ }^{29}$, qui était effectuée auprès d'un échantillon de 75 techniciens et agents de maîtrise, avait analysé les relations entre la nature de l'attachement d'un employé à l'égard de son organisation et la performance individuelle au travail. Les résultats de cette étude avaient établi l'existence de relations positives entre certaines dimensions de l'implication organisationnelle et la performance dans la tâche. En revanche, le degré d'association est sensiblement différent selon la nature de l'attachement.

- En 2020, l'étude de Karim, Komat et Koubaa ${ }^{30}$, qui était effectuée auprès d'un échantillon de 107 grandes entreprises opérantes au Maroc, avait comme objectif de relever les associations simultanées entre les systèmes $\mathrm{RH}^{31}$, les stratégies d'affaire et les deux indicateurs de la performance «sociétale/environnementale ${ }^{32}$. En se basant sur les principes de l'approche configurationnelle, cette étude avait montré que la cohérence interne entre les systèmes RH, ainsi que leur cohérence externe avec la stratégie d'affaires, peuvent constituer un important levier d'amélioration de la performance sociétale/environnementale.

Pour clôturer notre article, nous allons reprendre la station d'El Adraoui H. : «En dépit de nombreuses années de recherche, la question du lien RH-performance est loin d'être tranchée. Le regard porté par les managers marocains sur le lien RH-Performance est encore hésitant. Pour une raison simple : ils ne le maitrisent pas ${ }^{33}$.

\section{BIBLIOGRAPHIE}

[1] Azouzou H. : «La dimension culturelle de la qualité », Albayane, le 05 - 01 - 2014.

[2] Belalia A. : «Existe-t-il un management marocain ? », L’Economiste, Edition N 4398, Le 12/11/2014

[3] Bellal E. : «L'évolution de la gestion des ressources humaines au Maroc », colloque maghrébin sur les Systèmes de formation, stratégies d'entreprises et valorisation des ressources humaines; Faculté de droit de Rabat, 1994.

[4] Benabdeljlil N. : «Les modes de management des entreprises au Maroc : entre contingences culturelles et économiques », Revue internationale P.M.E., Vol. 20, N² 2, 2007, pp. 89-122.

\footnotetext{
${ }^{29}$ Yassine A. et Côme T. : «La nature de l'attachement entre l'employé et l'organisation, vecteur de la performance individuelle ?», Revue des Etudes Multidisciplinaires en Sciences Economiques et Sociales, Vol. 4, No 1, 2019.

${ }^{30}$ Karim S., Komat A. et Koubaa S. : «Les systèmes de gestion des ressources humaines et la performance sociétale/environnementale de la grande entreprise au Maroc », XXIXe Conférence Internationale de Management Stratégique, Juin 2020.

${ }^{31}$ Le système RH était conçu sous forme d'un ensemble de pratiques RH cohérentes entre elles

${ }^{32}$ La performance sociétale/environnementale était mesurée à travers l'« engagement sociétal » et la " présence d'une stratégie environnementale ».

${ }^{33}$ El Adraoui H., Professeur à l'ISCAE et consultante en RH : selon une étude sur le lien RH-Performance menée auprès de 92 entreprises nationales et multinationales au Maroc « GRH : Un toolkit pour les bonne pratiques » publiée dans l'Economiste du 14.12.2017.
} 
[5] Benabdeljlil N. : «Rapports sociaux et attitudes au travail dans les entreprises marocaines : entre contingences culturelles et contingences économiques », In Dupret et al. (dir), Le Maroc au présent, Ed. Dialogue des deux rives, 2015, pp. 985-994.

[6] Chaouki F. et Messoudi A. : «Les ressources humaines dans le modèle de performance des PME. Etude des Difficultés et des Besoins d'amélioration de la GRH dans les entreprises marocaines de petite et moyenne taille », Journal Ouest-Africain des Sciences de Gestion Vol.1, N², 2016, pp. 168-192.

[7] DIORH : «Enquête sur la fonction Ressources Humaines au Maroc », DIORH, Casablanca, 2002, 2004.

[8] Dlimi S. et Alami F-Z. : «Performance des équipes : cas des PME marocaines », Editions L'Harmattan, 2018.

[9] El Adraoui H. : «Effets de la GRH sur la performance: validation du modèle configurationnel auprès d'un échantillon de 92 entreprises au Maroc », Question (s) de management, Vol. 3, 2015, pp. 43-56.

[10] El Adraoui H. : «GRH : Un toolkit pour les bonne pratiques », L’Economiste du 14.12.2017.

[11] El Amrani J. et Chebihi M.A., «Culture et management au Maroc », Repères et perspectives, Rabat, n 4, hiver, 2003, p. 87107.

[12] El Asri L. : «Les pratiques de la gestion des ressources humaines : cas des grandes entreprises au Maroc », International Journal of Innovation and Scientific Research, Vol. 20, N² 2, Feb. 2016, pp. 453-463.

[13] Frimousse S. et Peretti J-M. : «Apprentissage Stratégique Des Pratiques De GRH, Internationalisation Des Firmes Et Espace Euromaghrébin », 2005.

[14] Hofstede G. : « Vivre dans un monde multiculturel : comprendre nos programmations mentales », Les Editions d'Organisation, Paris 1994

[15] Huteau S. : « Le management public territorial », Editions du Papyrus Montreuil, 2007.

[16] Guergachi A., Gueye A., Bentaleb C. et Ferar D. : « Le management des ressources humaines au Maroc, quelles pratiques performantes pour les entreprises marocaines? », A ctes du Colloque international La Gestion des Ressources Humaines dans les PME marocaines : quelles pratiques pour la performance ?, Casablanca, 15 et 16 Mars 2013.

[17] Karim S., Komat A. et Koubaa S. : «Les systèmes de gestion des ressources humaines et la performance sociétale/environnementale de la grande entreprise au Maroc », XXIXe Conférence Internationale de Management Stratégique, Juin 2020.

[18] Laalou A. et El Guermai L. : « Mesurer l'impact du Management des Ressources Humaines sur la Performance de l'entreprise : Cas de la société Source Marrakech », International Journal of Innovation and Applied Studies, Vol. 13, N², 2015.

[19] Maghni A. et Amine A. : « Socialisation Organisationnelle Et Engagement Des Nouvelles Recrues D’une Banque Marocaine : Etude Des Effets Spécifiques Par La Méthode PLS », Revue Marocaine de Management Logistique et Transport, N², 2017, pp. 239-272.

[20] Moutaouakil A. : «L'impact du Contrôle de Gestion Sociale sur la Performance Economique : Cas des Grandes Entreprises Marocaines », Thèse pour l'obtention de Doctorat en Sciences Economiques et Gestion, soutenue en 2015 à la FSJES Université Sidi Mohamed Benabdellah - Fès.

[21] Rafai A. : « La gestion des ressources humaines dans la fonction publique au Maroc », Edition 2013.

[22] Yassine A. et Côme T. : «La nature de l'attachement entre l'employé et l'organisation, vecteur de la performance individuelle? », Revue des Etudes Multidisciplinaires en Sciences Economiques et Sociales, Vol. 4, N 1, 2019. 